CLINICAL STUDY

\title{
A novel homozygous splice acceptor site mutation of KISS1R in two siblings with normosmic isolated hypogonadotropic hypogonadism
}

\author{
M G Teles ${ }^{1,4}$, E B Trarbach ${ }^{1}$, S D Noel ${ }^{4}$, G Guerra-Junior ${ }^{2}$, A Jorge ${ }^{1}$, D Beneduzzi ${ }^{1}$, S D Bianco ${ }^{4,6}$, A Mukherjee ${ }^{4,5}$, \\ M T Baptista ${ }^{2}$, E M Costa ${ }^{1}$, M De Castro ${ }^{3}$, B B Mendonça ${ }^{1}$, U B Kaiser ${ }^{4}$ and A C Latronico ${ }^{1}$ \\ ${ }^{1}$ Unidade de Endocrinologia do Desenvolvimento, Laboratório de Hormônios e Genética Molecular/LIM42 da Disciplina de Endocrinologia do Hospital das \\ Clínicas da Faculdade de Medicina da Universidade de São Paulo, 05403-900 São Paulo, Brazil, ${ }^{2}$ Departamento de Pediatria e Departamento de Clínica \\ Médica, Disciplina de Endocrinologia e Metabologia da Faculdade de Ciências Médicas da Universidade de Campinas, 10083-970 São Paulo, Brazil, \\ ${ }^{3}$ Departamento de Medicina Interna, Divisão de Endocrinologia, Faculdade de Medicina de Ribeirão Preto da Universidade de São Paulo, 14049-900 São \\ Paulo, Brazil, ${ }^{4}$ Division of Endocrinology, Diabetes and Hypertension, Brigham and Women's Hospital and Harvard Medical School, Boston, O2115 \\ Massachusetts, USA, ${ }^{5}$ Genes and Development/Reproduction, Royal Veterinary College, London, NW1 OTU UK and ${ }^{6}$ Department of Pharmacology, \\ University of Miami School of Medicine, Miami, 33136 Florida, USA
}

(Correspondence should be addressed to M G Teles; Email: milena.teles@gmail.com; or A C Latronico; Email: anacl@usp.br)

\begin{abstract}
Context: Loss-of-function mutations of the kisspeptin-1 receptor gene, KISS1R, have been identified in patients with normosmic isolated hypogonadotropic hypogonadism (nIHH).

Objective: To investigate KISS1R defects in patients with absent or delayed puberty.

Patients: We investigated KISS1R gene defects in a cohort of 99 Brazilian patients with nIHH or constitutional delay of puberty (CDP).

Methods: The entire coding region of KISS1R was amplified by PCR followed by automatic sequencing. In addition, screening for KISS1R exonic deletions was performed by multiplex ligation-dependent probe amplification.

Results: One novel homozygous KISS1R mutation was identified in two siblings with nIHH. This variant was an insertion/deletion (indel) mutation characterized by the deletion of three nucleotides (GCA) at position -2 to -4 , and by the insertion of seven nucleotides (ACCGGCT) at the same position, within the $3^{\prime}$ splice acceptor site of intron 2 of KISS1R. The brothers who carried this KISS1R mutation had no clinical evidence of pubertal development at the ages of 14 and 20 years. Computational analysis of this indel mutation predicted the generation of an abnormal protein. In addition, a new heterozygous KISS1R variant (p.E252Q) was identified in a male patient with sporadic nIHH. However, in vitro studies of this variant did not demonstrate functional impairment. Only known polymorphisms were identified in patients with CDP.

Conclusion: Loss-of-function mutations of KISS1R represents a rare cause of nIHH, and was absent in patients with CDP. We have described a novel KISS1R homozygous splice acceptor site mutation in the familial form of nIHH.
\end{abstract}

European Journal of Endocrinology 163 29-34

\section{Introduction}

The selective failure of gonadal function secondary to deficient pituitary gonadotropin secretion results in a clinical syndrome defined as isolated hypogonadotropic hypogonadism (1). The biological hallmark of this disorder is a decreased level of sex steroids associated with low or normal levels of FSH and LH without other pituitary hormonal deficiencies $(1,2)$.

In late 2003, loss-of-function mutations in KISS1R, also called GPR 54, were first described in two consanguineous families with hypogonadotropic hypogonadism and normal olfaction, as well as in three sporadic hypogonadotropic patients, by two independent groups $(3,4)$. More recently, a few additional inactivating mutations have been described in KISS1R in patients with sporadic normosmic isolated hypogonadotropic hypogonadism (nIHH) $(5,6)$. Interestingly, mice bearing homozygous target disruptions of this gene (Kiss1r-/-) exhibited a similar phenotype to that described in the patients harboring mutations (3, 7). These findings indicated that KISS1R and its cognate ligand, kisspeptin-1, are required for normal function of the hypothalamic-pituitary-gonadal axis, with an 
important role in the physiologic regulation of puberty $(3,8)$. Indeed, the relevance of the kisspeptin system in human puberty onset was also highlighted by the recent identification of a unique nonconstitutively activating mutation of the KISSIR gene in a girl with central precocious puberty (9). Here, we investigated KISS1R gene defects in a cohort of Brazilian patients with normosmic IHH or constitutional delay of puberty (CDP).

\section{Subjects and methods}

\section{Subjects}

We studied 69 Brazilian patients (43 males and 26 females) with $\mathrm{nIHH}$ and 30 patients ( 25 boys and 5 girls) with CDP. Nine patients with nIHH and ten patients with CDP had a history of other affected members in their families. Written informed consent was obtained from all patients. The collection of blood samples for genetic studies and the clinical protocols were approved by the Ethical Committee of the Hospital das Clínicas da Faculdade de Medicina da Universidade de São Paulo, São Paulo, Brazil. Chronic diseases were ruled out in these subjects.

IHH was defined as inappropriately normal or low gonadotropin levels together with prepubertal concentrations of sex steroids and absence of other pituitary hormonal deficiencies. All patients were normosmic, as assessed by smell tests (Smell Identification Test or Alcohol Sniff Test) (10). Magnetic resonance imaging appearances of the pituitary, hypothalamus, and olfactory bulbs were normal in all patients.

CDP was defined by lack of breast development by the age of 13 years in girls and lack of testicular enlargement in boys by the age of 14 years (11). All the 30 patients with CDP were followed until they were over 18 years. They exhibited slow progression of secondary sexual characteristics and delayed bone age. Pubertal development was completely achieved either spontaneously or after a brief course of sex steroid therapy.

The control population was derived from a Sao Paulo Clinical Hospital cohort of Brazilian subjects. One hundred and twenty unrelated individuals (age ranging from 18 to 56 years) who experienced normal pubertal development at the appropriate chronological age were used as controls. Pubertal development was addressed by a questionnaire that included the age at first signs of puberty, age of menarche for women, and a history of fathering or giving birth to child.

\section{DNA analysis of KISS1R}

Genomic DNA was extracted from peripheral blood leukocytes, and the five coding exons of KISS1R (Genbank accession no. - AY253981), including exon-intron boundaries, were amplified using Platinum TaqPCRx DNA Polymerase (Invitrogen Life Technologies) and intronic primers, as described previously (3).

All amplified fragments were examined on 1\% agarose gel electrophoresis. The PCR products were pretreated with an enzymatic combination of exonuclease I and shrimp alkaline phosphatase (United States Biochemical Corp., Cleveland, OH, USA), and directly sequenced using the BigDye terminator cycle sequencing ready reaction kit in an ABI PRISM 3100 automatic sequencer (Perkin Elmer Cetus, Waltham, MA, USA). All genetic variants were confirmed by automated sequencing using forward and reverse primers of two distinct PCR products. Computational splice site prediction was performed using NetGene2 software (http://genome.cbs. $\mathrm{dtu} . \mathrm{dk} /$ services/NetGene2/).

\section{Multiplex ligation-dependent probe amplification assay}

The multiplex ligation-dependent probe amplification assay (MLPA) is a recent method for relative quantification of 30-40 different DNA sequences in a single reaction. It has been used successfully for the detection of deletions and duplications in cell lines and tumor samples (12). MLPA was performed on genomic DNA obtained from all patients with IHH using SALSA MLPA kit P133 Kallmann-2 (MRC Holland), containing probes for FGFR 1 (exons 1-3, 5, 6, 8, 10, 11, 13, and 14), KISS1R (exons 1 and 4), GNRH1 (all three exons), GNRHR (all three exons), and NELF (exons 5, 11, and 15). Briefly, genomic DNA (20-500 ng) isolated from blood leukocytes was denatured at $98^{\circ} \mathrm{C}$ for $5 \mathrm{~min}$ and hybridized to MLPA probes according to the manufacturer's protocol. PCR was performed on a thermal cycler, and separation and detection of amplification products were performed by capillary electrophoresis on an ABI 310 DNA Genetic Analyzer (Applied Biosystems). Analysis was carried out by simple visual examination of the capillary electrophoresis peak profiles generated by the GeneScan Software (Applied Biosystems) compared with the control sample. Genetic dosage quotients near 1.0 are described as disomic.

\section{Generation of E252Q KISS1R by site-directed mutagenesis}

The E252Q KISS1R mutant was generated by site-directed mutagenesis with the QuikChange II Site-Directed Mutagenesis Kit (Stratagene, La Jolla, CA, USA), using the mammalian expression vector pCMVsport 6 containing the full-length wild-type (WT) human KISS1R as template (9). The presence of the mutation was confirmed by direct sequencing. 


\section{Inositol phosphate assay}

COS-7 cells were transiently transfected with $100 \mathrm{ng}$ WT KISS1R (WT), E252Q KISS1R, or empty vector (EV) (pCMVsport6) using GenePORTER Transfection Reagent (Gene Therapy Systems, San Diego, CA, USA). Measurement of total inositol phosphate (IP) production was performed $1 \mathrm{~h}$ following stimulation with increasing concentrations of kisspeptin as previously described (13). In brief, $24 \mathrm{~h}$ after transfection with WT or E252Q KISS1R or pCMVsport6, media on transfected COS-7 cells were replaced with inositol-free DMEM for $2 \mathrm{~h}$ at $37{ }^{\circ} \mathrm{C}$, after which $2 \mu \mathrm{Ci} / \mathrm{ml}$ myo- $\left[2-{ }^{3} \mathrm{H}\right]$-inositol (Perkin Elmer) was added, followed by the addition of $10 \mathrm{mM} \mathrm{LiCl} 15 \mathrm{~min}$ later. After overnight incubation, IP production was stimulated by the addition of $10^{-10}-10^{-6} \mathrm{M}$ kisspeptin-10 for $1 \mathrm{~h}$. Cells were extracted with formic acid, lysates were neutralized and centrifuged, and protein content was determined. Supernatants were loaded onto previously equilibrated AG-X8 resin anion exchange columns (Fisher Scientific, Pittsburgh, PA, USA). The columns were washed, and total IP was eluted. Radioactivity was quantified by liquid scintillation and corrected for protein content. All assay points were performed in triplicate, and each experiment was repeated at least three times.

\section{Results}

\section{DNA analysis of KISS1R}

Automatic sequencing of KISSIR revealed the presence of two new variants in three patients with IHH. The first variant was an insertion/deletion (indel) mutation characterized by the deletion of three nucleotides, GCA, at position -2 to -4 , and by the insertion of seven nucleotides, ACCGGCT, at the same position, within the $3^{\prime}$ splice acceptor site of intron 2 of KISS1R. This complex IVS2 -4_-2delGCAinsACCGGCT mutation was identified in the homozygous state in two male siblings with nIHH (Fig. 1). These two brothers had no clinical evidence of any pubertal development at the ages of 14 and 20 years. At birth, both had presented with micropenis and cryptorchidism, and exhibited prepubertal testosterone levels associated with low basal gonadotropins (patients 1 and 2, Table 1). The oldest brother underwent a GNRH stimulation test, and had prepubertal gonadotropin levels in response (patient 1, Table 1). Their mother carried the same mutation in the heterozygous state and reported normal pubertal development. Their father is believed to have had normal reproductive maturation and function, but was not available for testing.

The second KISS1R variant identified was characterized by a c. $754 \mathrm{G}>\mathrm{C}$ transversion in exon 5 , resulting in the substitution of glutamic acid by glutamine (p.E252Q) in the third intracellular loop of the receptor. This change was identified in the heterozygous state in a
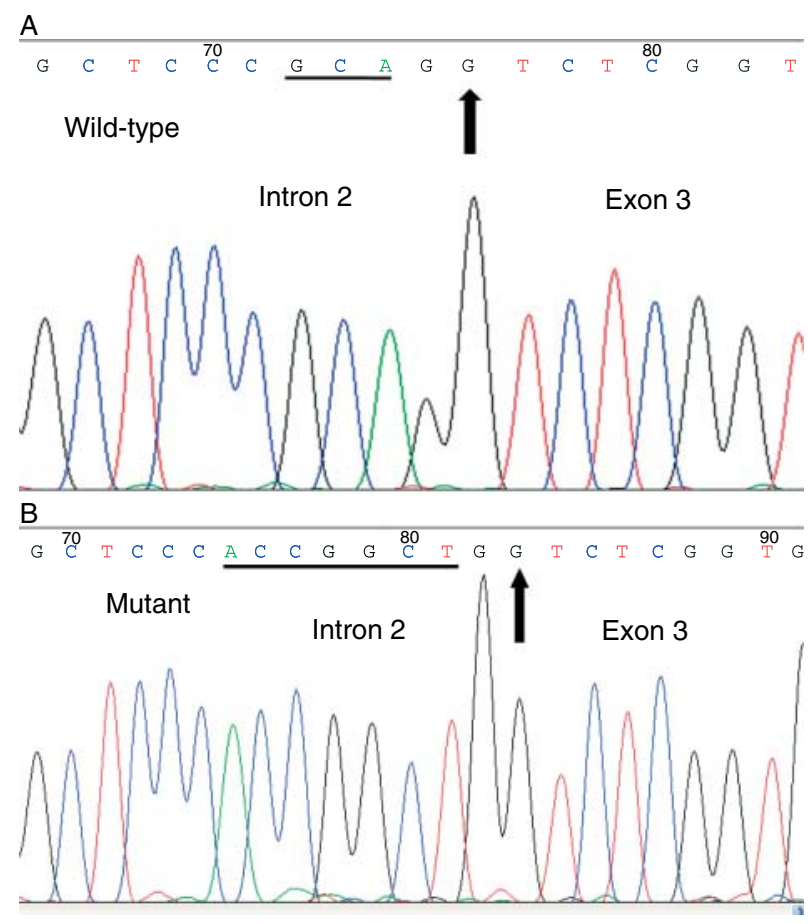

Figure 1 (A) KISS1R wild-type sequence in a normal control. The arrow indicates the beginning of exon 3. The nucleotides 'GCA' of the wild-type sequence of intron 2 are underlined. (B) Homozygous indel KISS1R mutation, IVS2 -2_-4 delGCA insACCGGCT, in a patient with familial IHH. The arrow indicates the beginning of exon 3 . The nucleotides 'GCA' of the wild-type sequence of intron 2 were substituted by the nucleotides underlined 'ACCGGCT' immediately before the beginning of exon 3 .

male patient with sporadic nIHH, who exhibited micropenis and cryptorchidism at birth (patient 3, Table 1). DNA analysis of his mother did not reveal the p.E252Q mutation. His father was not available for molecular studies.

These two KISS1R variants identified in this study were absent in a control group composed of 120 ethnically matched individuals. Previous analyses of FGFR1, GNRHR, GNRH, PROK2, PROKR2, TAC3, TACR3, and KISS1 genes did not reveal any additional genetic abnormalities in the patients carrying these KISS1R mutations. Six polymorphisms were also found, five of them previously described (Table 2). No KISS1R mutations were found in the group of patients with CDP.

\section{MLPA analysis}

KISS1R deletions involving exons 1 and 4 of the KISS1R gene were ruled out by MLPA study in the patient who carried the KISS1R p.E252Q variant. Additionally, exonic deletions of the FGFR1, GNRH1, GNRHR, and NELF genes were not detected using MLPA in this patient nor in the remaining group with nIHH. 
Table 1 Clinical and hormonal characteristics of three patients with normosmic isolated hypogonadotropic hypogonadism associated with KISS1R mutations.

\begin{tabular}{|c|c|c|c|c|c|c|c|c|c|}
\hline \multirow[b]{2}{*}{ Case } & \multirow{2}{*}{$\begin{array}{c}\text { Age } \\
\text { (years) }\end{array}$} & \multirow[b]{2}{*}{ Sex } & \multicolumn{2}{|c|}{ LH (IU/I) } & \multicolumn{2}{|c|}{ FSH (IU/I) } & \multirow{2}{*}{$\begin{array}{l}\text { Testo } \\
\text { (ng/dl) }\end{array}$} & \multirow[b]{2}{*}{ KISS1R mutations } & \multirow[b]{2}{*}{ State } \\
\hline & & & Basal & Peak & Basal & Peak & & & \\
\hline $1^{\mathrm{a}}$ & 20 & $M$ & 0.37 & 4.34 & 2.3 & 6.39 & 24 & $\begin{array}{l}\text { IVS2 -2_-4 delGCA } \\
\text { insACCGGCT }\end{array}$ & Homozygous \\
\hline $2^{a}$ & 14 & M & $<0.1$ & - & 1.2 & - & 24 & $\begin{array}{l}\text { IVS2 -2_-4 delGCA } \\
\text { insACCGGCT }\end{array}$ & Homozygous \\
\hline 3 & 18 & M & 0.7 & - & 2.4 & - & 20 & p.E252Q & Heterozygous \\
\hline
\end{tabular}

M, male; Testo, testosterone. Hormonal levels measured by electrochemiluminescence. LH sensitivity, 0.1 IU/l; FSH sensitivity, 0.1 IU/I; testosterone sensitivity, $2 \mathrm{ng} / \mathrm{dl}$.

apatients 1 and 2 are brothers.

\section{Computational splice site prediction}

The splice site KISS1R mutation (IVS2 -4_-2delGCAinsACCGGCT) was analyzed by NetGene2 software. This mutation is predicted to have the potential to generate four different abnormal KISS1R proteins using alternative acceptor sites (Fig. 2). The use of sites 1 and 4 results in truncated proteins after the second intracellular and second extracellular loops respectively. The use of site 2 results in a longer 410-amino acid protein, while the use of site 3 results in a receptor lacking four conserved amino acids (QVSV).

\section{Computational E252Q variant prediction}

We applied two in silico algorithms, the PolyPhen algorithm (http://genetics.bwh.harvard.edu/cgi-bin/ ggi/ggi.cgi) and the SIFT algorithm (http://sift.jcvi.org) to predict the putative effect of the E252Q amino acid substitution on KISS1R function. This substitution was predicted to be tolerated (benign) using both SIFT and PolyPhen algorithms.

\section{Functional analysis of the E252Q variant}

IP production was measured in COS-7 cells transiently transfected with WT or E252Q KISS1R or EV, and the response to stimulation with increasing concentrations $\left(10^{-10}-10^{-6} \mathrm{M}\right)$ of human kisspeptin-10 was measured. Kisspeptin dose-response curves were similar for WT and E252Q KISS1R, with no notable loss of activity of the E252Q mutant (Fig. 3). Cells transfected with EV failed to show any IP induction in response to kisspeptin-10 treatment (not shown).

\section{Discussion}

Since the first description in 2003, eight different lossof-function mutations have been described in the KISS1R gene in patients with nIHH. These mutations include six point mutations (p.L148S, p.R297L, p.C223R, p.R331X, p.X399R, and p.L102P) (3, 5, $14)$, one 155-bp deletion (4), and one insertion
(c.1001_1002insC) (6). Taking into consideration the 312 patients with normosmic IHH reported to have been screened, including the 69 patients from this study, mutations reported in KISS1R gene are responsible for $<5 \%$ of cases of normosmic IHH. Therefore, inactivating KISS1R mutations have been shown to be responsible for only a small number of familial and, even less commonly, sporadic cases of normosmic hypogonadotropic hypogonadism.

Patients with inactivating KISS1R mutations present with delayed puberty and no apparent associated conditions. Micropenis and cryptorchidism are described in some cases, suggesting that the kisspeptin-KISS1R system may play an important role in GNRH secretion during fetal development in utero $(3-6,14)$. The acute response to GNRH stimulation was variable among patients with KISS1R mutations and ranged from totally blunted to normal. The present report describes two novel variants in KISS1R gene. The first variant was identified in two siblings with isolated hypogonadotropic hypogonadism who presented with micropenis and cryptorchidism at birth. The alteration is a homozygous indel mutation characterized by a deletion of three nucleotides at position -2 to -4 and by an insertion of seven nucleotides (IVS2 -2_-4delGCAinsACCGGCT) in the same position in the $3^{\prime}$ splice acceptor site of intron 2. This mutation was absent in 240 alleles of normal individuals. Although experimental studies are necessary to fully characterize the effect of this mutation, single nucleotide changes in the canonical AG/GT nucleotides at the splice junctions have been described in several disorders (15).

Table 2 Prevalence of exonic variants in KISS1R gene in the 69 isolated hypogonadotropic hypogonadism patients.

\begin{tabular}{llll}
\hline dbSNP ID & SNP & Amino acid & Prevalence (\%) \\
\hline ss14700521 & c.24A $>$ G & p.G08G & 23.6 \\
- & c.83G $>$ C & p.S61S & 13.1 \\
rs350132 & c.1091T>A & p.L364H & 34.2 \\
- & c.565 G >A & p.A189T & 5.2 \\
rs8111938 & c.615 A >C & p.A205A & 2.6 \\
\hline
\end{tabular}

${ }^{\mathrm{a}} \mathrm{A}$ new variant. 


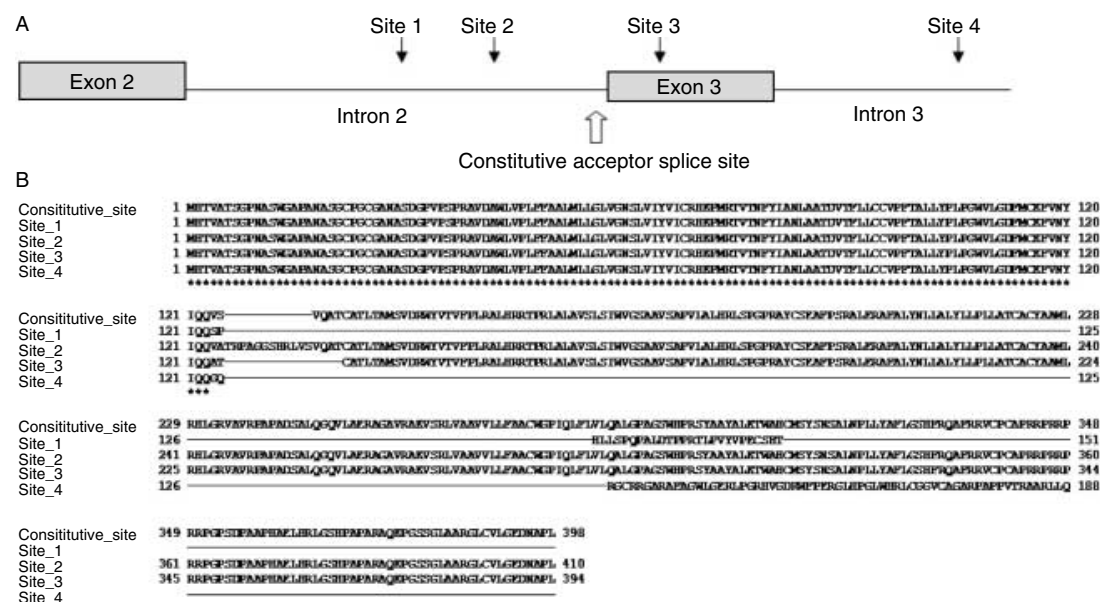

Figure 2 (A) Schematic representation of exons 2 and 3 of $K I S S 1 R$ gene. The white arrow indicates the constitutive acceptor splice site within intron 2; the four black arrows indicate the cryptic sites which could be used after the loss of the constitutive acceptor splice site. (B) Alignment of the 398 amino acids of wild-type KISS1R and the four abnormal predicted proteins. The use of sites 1 and 4 results in truncated proteins with 151 and 188 amino acids respectively. The use of site 2 results in a longer protein of 410 amino acids, whereas the use of site 3 results in a protein lacking four highly conserved amino acids (QVSV).
Computational splice site prediction confirms that the substitution of the GCA nucleotides by ACCGGCT in the KISS1R mutated patients could result in aberrant intron retention or exon 3 skipping due to disruption of the constitutive sites and the use of cryptic splice acceptor sites. Both events would be predicted to result in the synthesis of severely truncated receptors, based on the introduction of premature stop codons or the insertion/deletion of different residues in the protein (Fig. 2). We hypothesize that this novel KISS1R indel mutation leads to the production of abnormal transcripts and therefore to receptor loss-of-function.

The p.E252Q variant was identified in the heterozygous state in a male with sporadic IHH. The glutamic acid at position 252 is conserved in pig, zebrafish, and urchin, but not in primate, mouse, or rat. This KISS1R missense variant was also absent in 240 alleles of ethnically matched controls. The E252Q variant was predicted to be benign using both SIFT and PolyPhen algorithms. Surprisingly, no other KISS1R exonic mutation or deletion was identified in this patient, except for known polymorphisms. In addition, FGFR1, GNRHR, GNRH, PROK2, PROKR2, TAC3, TACR3, and KISS1 mutations were not detected in this patient. All previously described KISS1R mutations associated with patients with $\mathrm{IHH}$ are in the homozygous or compound heterozygous state, suggesting an autosomal recessive mode of inheritance for this condition. As we examined primarily the coding sequence in this study, it is certainly possible that the patient carries a variant in the non-coding regions not analyzed, such as introns or regulatory regions. We cannot rule out the possibility that the p.E252Q variant represents a rare and novel polymorphism which does not have a functional effect on KISS1R, since the in vitro studies of this variant were not able to demonstrate functional impairment.

CDP is the most common cause of delayed puberty within the general population (16-18). It frequently aggregates in families, suggesting that it is genetically determined (19). The most frequent pattern of inheritance described in patients with CDP is autosomal dominant; however, an autosomal recessive pattern of inheritance has been described in $\sim 2-4 \%$ of CDP cases (11). Since KISS1R mutations constitute a rare cause of autosomal recessive $\mathrm{HH}$, we hypothesized that variations in this gene could be associated with the unusual autosomal recessive form of CDP and less severe KISS1R mutations. However, in this study, no KISS1R mutations were identified in this group of patients, in agreement with a previous publication (5).

In conclusion, we have described a complex KISS1R splice site mutation in two patients with IHH. This indel mutation potentially impairs KISS1R function leading to the nIHH phenotype.

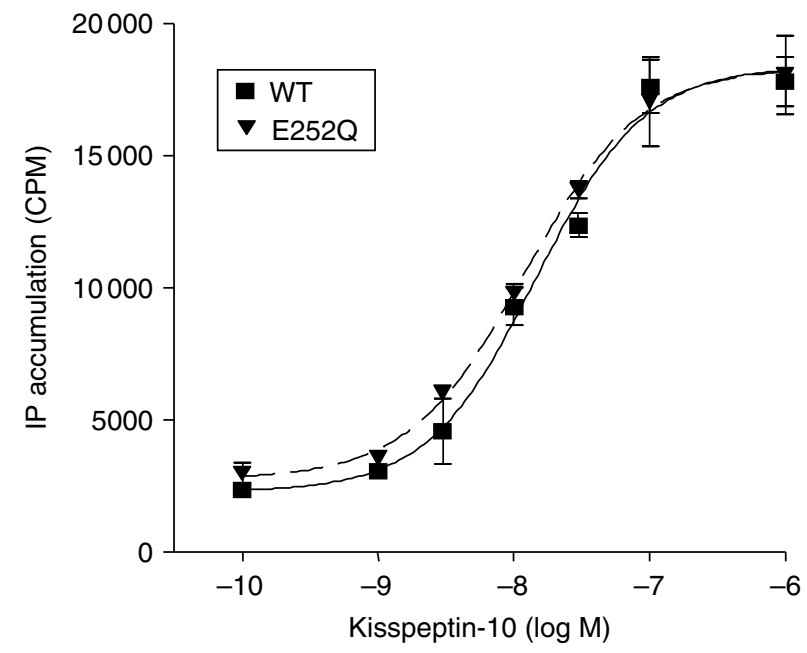

Figure 3 Kisspeptin stimulation of inositol phosphate (IP) accumulation by wild-type (WT) and E252Q mutant KISS1R. IP accumulation was measured in COS-7 cells transiently transfected with cDNA encoding WT or E252Q KISS1R. Dose-response analysis was performed by stimulating cells for $1 \mathrm{~h}$ with increasing concentrations of human kisspeptin- $10\left(10^{-10}-10^{-6} \mathrm{M}\right)$. Data points represent the mean \pm S.E.M. of triplicate samples. The figure is a representative graph from three independent experiments. 


\section{Declaration of interest}

The authors declare that there is no conflict of interest that could be perceived as prejudicing the impartiality of the research reported.

\section{Funding}

This work was supported in part by grants from Fundação de Amparo Estado de São Paulo - FAPESP, processos \# 05/04726- (A C L and M G T) and 0550146-5 (M G T) and by the Eunice Kennedy Shriver NICHD/NIH through cooperative agreement U54 HD28138 as part of the Specialized Cooperative Centers Program in Reproduction and Infertility Research (U B K).

\section{References}

1 Hoffman AR \& Crowley WF Jr. Induction of puberty in men by long-term pulsatile administration of low-dose gonadotropinreleasing hormone. New England Journal of Medicine $1982 \mathbf{3 0 7}$ 1237-1241.

2 Hayes FJ, Seminara SB \& Crowley WF Jr. Hypogonadotropic hypogonadism. Endocrinology and Metabolism Clinics of North America 199827 739-763 vii.

3 Seminara SB, Messager S, Chatzidaki EE, Thresher RR, Acierno JS Jr, Shagoury JK, Bo-Abbas Y, Kuohung W, Schwinof KM, Hendrick AG, Zahn D, Dixon J, Kaiser UB, Slaugenhaupt SA, Gusella JF, O'Rahilly S, Carlton MB, Crowley WF Jr, Aparicio SA \& Colledge WH. The GPR 54 gene as a regulator of puberty. New England Journal of Medicine 2003349 1614-1627.

4 de Roux N, Genin E, Carel JC, Matsuda F, Chaussain JL \& Milgrom E. Hypogonadotropic hypogonadism due to loss of function of the KiSS1-derived peptide receptor GPR54. PNAS $200310010972-10976$.

5 Semple RK, Achermann JC, Ellery J, Farooqi IS, Karet FE, Stanhope RG, O'Rahilly S \& Aparicio SA. Two novel missense mutations in $\mathrm{g}$ protein-coupled receptor 54 in a patient with hypogonadotropic hypogonadism. Journal of Clinical Endocrinology and Metabolism 200590 1849-1855.

6 Lanfranco F, Gromoll J, von Eckardstein S, Herding EM, Nieschlag E \& Simoni M. Role of sequence variations of the GnRH receptor and G protein-coupled receptor 54 gene in male idiopathic hypogonadotropic hypogonadism. European Journal of Endocrinology 2005 $153845-852$.

7 Funes S, Hedrick JA, Vassileva G, Markowitz L, Abbondanzo S, Golovko A, Yang S, Monsma FJ \& Gustafson EL. The KiSS-1 receptor GPR54 is essential for the development of the murine reproductive system. Biochemical and Biophysical Research Communications 2003312 1357-1363.

8 Colledge WH. GPR54 and puberty. Trends in Endocrinology and Metabolism 200415 448-453.

9 Teles MG, Bianco SD, Brito VN, Trarbach EB, Kuohung W, Xu S, Seminara SB, Mendonca BB, Kaiser UB \& Latronico AC. A GPR54-activating mutation in a patient with central precocious puberty. New England Journal of Medicine 2008358 709-715.

10 Davidson TM \& Murphy C. Rapid clinical evaluation of anosmia. The alcohol sniff test. Archives of Otolaryngology - Head and Neck Surgery 1997123 591-594.

11 Sedlmeyer IL \& Palmert MR. Delayed puberty: analysis of a large case series from an academic center. Journal of Clinical Endocrinology and Metabolism 200287 1613-1620.

12 Schouten JP, McElgunn CJ, Waaijer R, Zwijnenburg D, Diepvens F \& Pals G. Relative quantification of 40 nucleic acid sequences by multiplex ligation-dependent probe amplification. Nucleic Acids Research 200230 e57.

13 Bedecarrats GY, Linher KD \& Kaiser UB. Two common naturally occurring mutations in the human gonadotropin-releasing hormone $(\mathrm{GnRH})$ receptor have differential effects on gonadotropin gene expression and on GnRH-mediated signal transduction. Journal of Clinical Endocrinology and Metabolism 2003 88 834-843.

14 Tenenbaum-Rakover Y, Commenges-Ducos M, Iovane A, Aumas C, Admoni O \& de Roux N. Neuroendocrine phenotype analysis in five patients with isolated hypogonadotropic hypogonadism due to a L102P inactivating mutation of GPR 54. Journal of Clinical Endocrinology and Metabolism 200792 1137-1144.

15 Buratti E, Baralle M \& Baralle FE. Defective splicing, disease and therapy: searching for master checkpoints in exon definition. Nucleic Acids Research 200634 3494-3510.

16 Rosenfield RL. Clinical review 6: diagnosis and management of delayed puberty. Journal of Clinical Endocrinology and Metabolism 199070 559-562.

17 Argente J. Diagnosis of late puberty. Hormone Research 199951 95-100.

18 Kulin HE. Delayed puberty. Journal of Clinical Endocrinology and Metabolism 199681 3460-3464.

19 Sedlmeyer IL, Hirschhorn JN \& Palmert MR. Pedigree analysis of constitutional delay of growth and maturation: determination of familial aggregation and inheritance patterns. Journal of Clinical Endocrinology and Metabolism 200287 5581-5586.

Received 25 March 2010

Accepted 28 March 2010 